\title{
Investigation of ANGPTL3 expression, exon sequence and promotor methylation between Ningxiang and Changbai pigs
}

\author{
Zhang DS ${ }^{1}$, Liu ZY ${ }^{1}$, Chen XJ', Kuang GW², Pan RT ${ }^{1}$, *Sun ZL ${ }^{1}$ \\ 1. College of Veterinary Medicine, Hunan Agricultural University, Changsha, Hunan 410128, China \\ 2. Institute of Hunan Province veterinary drugs and feed supervision, Changsha, Hunan 410006, China
}

\begin{abstract}
Background: Angiopoietin-like protein 3 (ANGPTL3), a member of angiopoietin-like proteins, has been demonstrated to affect lipid metabolism by inhibiting the activity of lipoprotein lipase (LPL).

Objective: To compare the ANGPTL3 mRNA and protein expression, exon mutation and promoter district CpG island methylation state between Ningxiang Pigs and Changbai Pigs.

Methods: Pigs were slaughtered and about $100 \mathrm{mg}$ of tissue samples and subcutaneous adipose tissue were collected and stored for analysis. Quantitative Real-Time PCR, Western blotting, exons sequencing, and HRM analysis were carried out. Results: ANGPTL3 was expressed in liver but not in fat and lean meat. Compared with Changbai pigs, ANGPTL3 mRNA level in Ningxiang pigs was lower. However, the protein expression showed no difference between these two groups of pigs. Sequences analysis showed that four variations existed between Changbai Pigs and Ningxiang Pigs, among which three variations caused no change of amino acids, and the other one caused amino acid mutation from Val (Changbai) to Met (Ningxiang). The ANGPTL3 promoter district CPG island bisulfite-PCR and sequencing results showed that the mean methylation rate ranged from $70.952 \%$ to $95.238 \%$ between Changbai pig and Ningxiang pig $(\mathrm{p}<0.05)$.

Conclusion: These results support the significant difference $(\mathrm{p}<0.05)$ between Changbai pig and Ningxiang pig in highresolution melting (HRM) analysis. All these results may be helpful for a better understanding of the role of ANGPTL3 in lipid metabolism.
\end{abstract}

Key words: Angiopoietin-like protein 3 (ANGPTL3), DNA methylation, pigs

African Health Sciences 2012; (4): 522 - 529 http://dx.doi.org/10.4314/ahs.v12i4.20

\section{Introduction}

Lipoprotein lipase (LPL), a water soluble enzyme catalyzing the hydrolysis of plasma triglycerides (TGs), plays a key role in lipid metabolism and transport of lipids. To date, the regulatory mechanisms on LPL expression have been widely investigated ${ }^{1}$, and angiopoietin-like 3 (ANGPTL3) has been demonstrated to play a key role in the regulation of LPL activity ${ }^{2}$. ANGPTL3 contains a signal peptide, an $\mathrm{N}$-terminal segment containing coiled-coil domain, and a C-terminal fibrinogen-like domain. The N-terminal segment as well as fulllength ANGPTL3 has been shown to inhibit LPL activity, and deletions of the N-terminal segment of ANGPTL3 might result in total loss of LPLinhibiting activity ${ }^{3,4}$. Previous studies have also shown ${ }^{5-}$

\section{*Corresponding author:}

Prof. Zhi-Liang Sun

Hunan Agricultural University

Furong District, Changsha 410128

Hunan, China

Tel: +86-731-84635229

E-mail: sunzhiliang1965@yahoo.com.cn
7 that ANGPTL3 exerts the main role in the regulation of lipid metabolism. Recent studies showed that either the injection of recombinant ANGPTL3 protein or adenovirus-mediated production of ANGPTL3 could acutely increased plasma triglyceride levels in both KK/San and wildtype lean mice 8 . The experiment carried out by Xiao 9. showed that ANGPTL3 contributed to the development of metabolic disorder, and 1,3,5,8tetrahydroxyxanthone (Xan) can regulates ANGPTL3-LPL pathway to lessen the ketosis in mice. The mRNA level of ANGPTL3 in liver tissue is indicated to be much higher in genetically obese pigs than that in lean counterparts ${ }^{10}$.

DNA methylation in the promoter regions plays a key role in silencing the genes in differentiated mammalian somatic cells ${ }^{11,12}$. In the process of DNA methylation, the methylated cytosine residues in the sequence 5'-CG-3' (so-called CpG motifs) within the genome of differentiated somatic cells is stable and clonally inherited ${ }^{13}$. Understanding the methylation of ANGPTL3 DNA will facilitate the understanding of the influence of ANGPTL3

African Health Sciences Vol 12 Issue 4 December 2012 
towards lipid metabolism.

The information on the exon sequencing and promoter $\mathrm{CpG}$ island methylation differences between the Ningxiang pigs (genetically obese pigs) and Changbai pigs (genetically lean pigs) remains to be unclear. Therefore, the aim of the present study is to decipher these informations, which may be helpful to a better understanding of the role of ANGPTL3 in lipid metabolism.

\section{Methods}

\section{Tissue preparation}

The collection of animal Tissues was approved by the Hunan Agricultural University College of Veterinary Medicine. After the pigs (Ningxiang pig weight about $75 \mathrm{~kg}$ and Changbai pig weight about $90 \mathrm{~kg}$ ) were slaughtered, approximately $100 \mathrm{mg}$ of tissue samples (the liver, muscle (lean meat, lean) and subcutaneous adipose tissue (fat meat, fat)) were immediately collected and stored at $-80^{\circ} \mathrm{C}$ until analysis.

\section{Quantitative Real-Time PCR}

Tissue samples $(20 \mathrm{mg}$ ) were homogenized in $1 \mathrm{ml}$ ice-cold Trizol reagent (Changsha Axybio bio-tech Co., LTD, Changsha, China) according to the manufacturer's instructions. Quality of RNA was verified with an Agilent 2100 Bioanalyzer (Agilent Technologies, Santa Clara, CA). To synthesize singlestranded cDNA from total RNA, we used the HighCapacity cDNA Reverse Transcription Kit (Fermentas, USA) with 1 ig of total RNA per 20 il reaction. Reverse transcription was performed on a thermal cycler(BioRad, Richmond, CA) by using the following protocol: $25^{\circ} \mathrm{C}$ for $10 \mathrm{~min}, 42^{\circ} \mathrm{C}$ for 60 min, $70^{\circ} \mathrm{C}$ for $10 \mathrm{~min}$, and cooling at $4^{\circ} \mathrm{C}$.

Relative ANGPTL3 expression was quantified by using the $2^{-\ddot{A} A \mathrm{Ct}}$ method with an ABI Prism 7500 real-time PCR Apparatus (Applied Biosystems). All sample values were normalized against GAPDH values. Primers were designed with Primer Express software (Applied Biosystems) using GenBank accession \#NM 001003926.1 for ANGPTL3 and accession \#NM 001206359.1 for porcine GAPDH. Primer Sequences were as follows: forward primer 5'-GATGGCTCCGTGGACTT'TAACC-3' and reverse primer 5'GGATGTGATGCACCTTCTCCAG-3' for ANGPTL3;

$$
\text { Forward primer 5' }
$$

GAACAAACGTGAGGTCTGGAGG-3' and reverse primer 5'-
CGTGGGTGGAATCATACTGG-3' for GAPDH; Both primer sets were designed to span an exon-exon boundary and melt curves $\left(60^{\circ} \mathrm{C}\right.$ to $95^{\circ} \mathrm{C}$ ) were monitored to verify that a single transcript was amplified. Amplification was performed in a 96-well plate with SYBR Premix Ex Taq Kit (Takara Bio USA, Madison, WI). Amplifications were performed in triplicate by using a standard shuttle PCR protocol $\left(30 \mathrm{~S} ; 15 \mathrm{~s}\right.$ at $95^{\circ} \mathrm{C}$ and $30 \mathrm{~s}$ at $60^{\circ} \mathrm{C}$, for 40 cycles).

Data were recorded and analyzed with Sequence Detector software (Applied Biosystems), and means and standard deviations were calculated for each tissue type.

\section{Western Blot analysis}

Liver, lean and fat were lysed for 30 minutes on ice in $20 \mathrm{mM}$ Tris-HCl (pH 7.50), $150 \mathrm{mM} \mathrm{NaCl}, 1 \%$ Triton, $1 \mathrm{mM} \mathrm{Na}{ }_{2}$ EDTA, $1 \mathrm{ig} / \mathrm{ml}$ leupeptin, $1 \mathrm{mM}$ phenylmethysulfonyl fluoride, $1 \mathrm{mM} \mathrm{Na} \mathrm{VO}_{4}, 1 \mathrm{mM}$ EGTA, $2.5 \mathrm{mM}$ sodium pyrophosphate, and $1 \mathrm{mM}$ betaglycerophosphate. Lysates and controls (Chemicon International, Temecula, CA) (30 ig/lane) were separated by sodium dodecyl sulfate polyacrylamide gel electrophoresis through a $12 \%$ TRIS-glycine gel, followed by transferring to nitrocellulose membranes (Bio-Rad, Hercules, CA). The membranes were blocked and incubated with antibody specific for rabbit ANGPTL3 (ab84035, 1:1, 000 dilution; Santa Cruz Biotechnology) and a rabbit anti-GAPDH control antibody ( 1:1, 000 dilution; Santa Cruz Biotechnology) and the respective secondary antibody (1:2, 000 dilution; Santa Cruz Biotechnology). Blots were developed with the ECLplus detection system (GE Healthcare, Waukesha, WI). And means and standard deviations were calculated for each tissue type.

\section{ANGPTL3 exons sequencing}

The genomic DNA was extracted with a Genomic DNA Purification Kit (Promega, Madison, WI, USA). All seven porcine ANGPTL3 exons were amplified by PCR, the primers were listed in table 1. The PCR products were run on 1.2\% agarose gel electrophoresis and purified, then sequenced by 3730 sequencer inNanjing Genescript bio-tech Co., LTD. 
Table 1: The primers for amplifying seven porcine ANGPTL3 exons

\begin{tabular}{ll}
\hline ANGPTL3-e1F & 5'-GCCAACCTTACTGTTTA-3' \\
ANGPTL3-e1R & 5'-AATGCTCTTTGGTTTCT-3' \\
ANGPTL3-e2F & 5'-GAAGCGTCTAACCAACC-3' \\
ANGPTL3-e2R & 5'-GTCCCTTTATTCACTATCC-3' \\
ANGPTL3-e3F & 5'-TTCCTCTTAACATCGGAAAC-3' \\
ANGPTL3-e3R & 5'-GCCATAGCGGTGACAAT-3' \\
ANGPTL3-e4F & 5'-GGCTACT'TCACCACAA-3' \\
ANGPTL3-e4R & 5'-CTTCAAAGCAAAGATAACA-3' \\
ANGPTL3-e5F & 5'-ACTACCTTACAAAGCCACC-3' \\
ANGPTL3-e5R & 5'-TTCTGCTGCTCCGTGAT-3' \\
ANGPTL3-e6F & 5'-GCAGTTAGCAACCCACA-3' \\
ANGPTL3-e6R & 5'-TTGGCTGATACTCAAGGA-3' \\
ANGPTL3-e7F & 5'-TATACTCATGCAGAAACATAG-3' \\
ANGPTL3-e7R & 5'-TTCTTCTAAAGCCTTCTATTA-3' \\
\hline
\end{tabular}

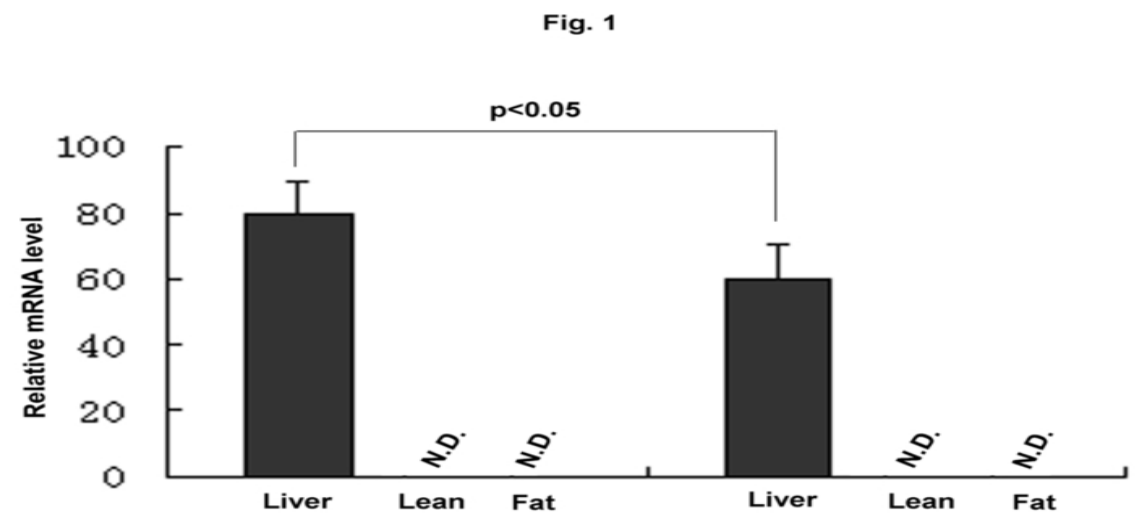

$279 \times 179 \mathrm{~mm}(72 \times 72 \mathrm{DPI})$

Figure 1: Investigation of mRNA levels of ANGPTL3 in different tissues using real-time PCR analysis. N.D, not detected. The experiments were performed in three replicates, and the data were given as Mean \pm S.D

BSP-Sequencing Analysis to promoter district CpG Island's Methylation Rate

To examine the DNA methylation patterns of the ANGPTL3 gene, we extracted genomic DNA with a Genomic DNA Purification Kit (Promega, Madison, WI, USA) and the DNA were treated with Epi Tect Bisulfite Kit (Qiagen, USA). In brief, 2 ìg of genomic DNA was denatured by treatment with $3 \mathrm{M} \mathrm{NaOH}$ and modified with $3 \mathrm{M}$ sodium bisulfite for $16 \mathrm{~h}$ at $42^{\circ} \mathrm{C}$ water bath. DNA samples were purified with the kit specification, and resuspended in 25 il water. We used PCR to get the target fragments, the primers were as follows:

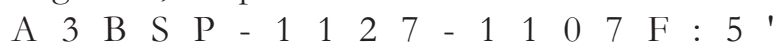
ATATGGAGGT'T'T'TAGGTTAG 3'
A 3 B S P $-8 \begin{array}{lllllllll} & 8 & 4 & - & 5 & 9 & \mathrm{R} & : & 5\end{array}$ AAAAATAAATATTCCACTATACTTC 3'

PCR product size was $268 \mathrm{bp}$, included $14 \mathrm{CpG}$ sites of the ANGPTL3 promotor district from the transcript start site -1127 to -860 bases.

The PCR program was as follows; $94^{\circ} \mathrm{C}$ 5 min; $94^{\circ} \mathrm{C} 30 \mathrm{~s}, 50^{\circ} \mathrm{C} 30 \mathrm{~s}, 72^{\circ} \mathrm{C} 30 \mathrm{~s}, 30$ cycles; $72^{\circ} \mathrm{C} 10 \mathrm{~min}$. The $\mathrm{PCR}$ product run on $1.5 \%$ agarose gel and the target fragments were purified with Wizard DNA Purification Resin (Promega) and cloned into pMD-18T vector (Takara). Five positive clones of each sample were sequenced in Shanghai Invitrogen Bio-tech Corporation. And the $\mathrm{CpG}$ island methylation rate was calculated as follows: $(\mathrm{C}$ number/(C number + T number $)) * 100 \%$. 
HRM Analysis to promoter district CpG Island's Methylation Rate

We used HRM to appraisal the BSP-Sequencing results reliability. That is, using the same primers and BSP DNA templates as BSP-Sequencing. The Standard samples were synthesized by Nanjing Genscript Bio-tech Co,. Ltd (Nangjing, China).

PCR amplification of the DNA was carried out using a Roche LightCycler 480 (Roche Applied Science, Laval, PQ, Canada) equipped with the Gene Scanning software (Version 1.5.0). PCR was performed in a 20il reaction volume and $2 \mathrm{ng}$ of BSP DNA templates ( 2 il) were added to each well which contained $1 \times$ Light Cycler 480 High Resolution Melting (HRM) Master Mix ${ }^{\circledR}$ (Roche), $3.0 \mathrm{mM} \mathrm{MgCl}_{2}$ and $0.2 \mathrm{iM}$ of each primer.

PCR amplification of the DNA was performed in a 20 il reaction volume, which contained 1× HRM PCR Master Mix (takara), 2 ng of BSP DNA templates and $0.2 \mathrm{iM}$ of each primer. PCR amplification was carried out using a Qiagen Rotor-Gene 6000 (Qiagen) at following conditions: $95^{\circ} \mathrm{C}$ for $10 \mathrm{~min}$, followed by 40 cycles at $95^{\circ} \mathrm{C}$ for $20 \mathrm{~s}, 58^{\circ} \mathrm{C}$ for $30 \mathrm{~s}$ and $72^{\circ} \mathrm{C}$ for $20 \mathrm{~s}$. To gain fluorescence for each cycling at $72^{\circ} \mathrm{C}$ requires the operator to choose the 'green' option. High resolution melting analysis is performed at ramp from $50^{\circ} \mathrm{C}$ to $90^{\circ} \mathrm{C}$, raising by $0.3^{\circ} \mathrm{C}$ each step, pausing $90 \mathrm{~s}$ at pre-melt condition as a first step and pausing $2 \mathrm{~s}$ for each step thereafter. To acquire melting fluorescence, 'green' needs be chosen. In silico computer graphics reveals the live PCR run, the concentration of PCR product at the stationary stage and the subsequent melt curves. First order differential plots of the melt curves of the PCR product are created by the software provided with the Rotor-Gene ${ }^{\mathrm{TM}} 6000$. Both peak-height and area-under-the-curve from the normalized, temperature-shifted, difference curves were used to generate a standard curve and determine the degree of methylation of each DNA sample.

\section{Statistical analysis}

Statistical analysis was performed and $\mathrm{P}$ values were calculated according to student's $t$ test. Significant differences were accepted for $\mathrm{p}<0.05$.

\section{Results}

Investigation of expression ANGPTL3 in different tissues of pigs

The results in figure 1 showed that the expression of ANGPTL3 was mainly restricted to liver but not in lean and fat, which is consistent with the situation in mice ${ }^{14}$. The mRNA level in the liver of Changbai pig was higher than that in Ningxiang pig $(\mathrm{p}<0.05)$. Similar to mRNA result, the protein expression of ANGPTL3 was also in liver but no in fat and lean (figure 2A). The ANGPTL3 expression level showed no differences in the liver between Changbai pig and Ningxiang pig ( $p>0.05$, figure $2 \mathrm{~B})$.

A

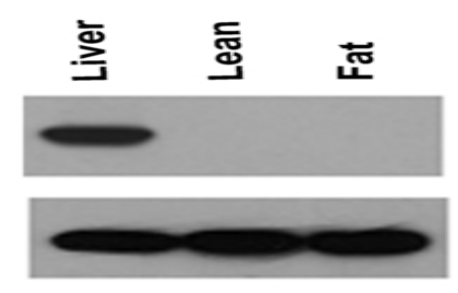

Changbai pig

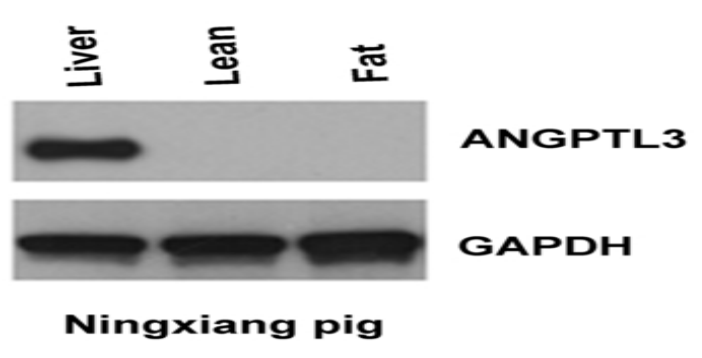

$\mathbf{B}$

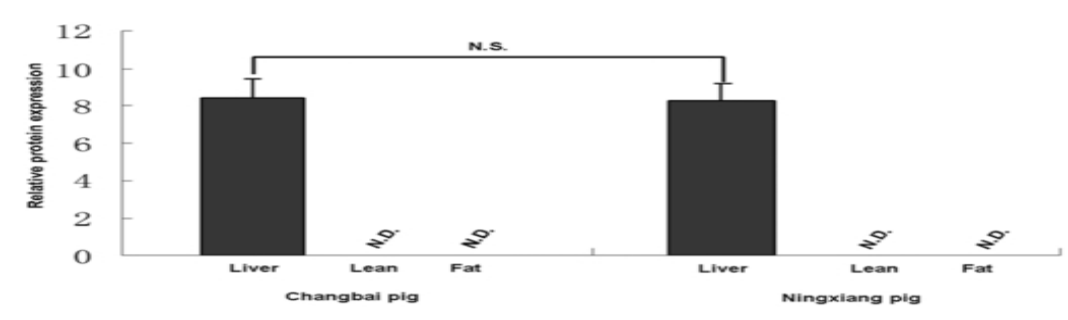

Fig. 2

Figure 2: Western blotting analysis of ANGPTL3 expression in different tissues. (A) Representative figure of western blotting. (B) Statistical analysis of ANGPTL3 expression.

N.S., not significant, N.D., not detected. The experiments were performed in three replicates, and the data were given as Mean \pm S.D. 


\section{Variants in ANGPTL3 Exons}

The ANGPTL3 exons sequence was analyzed for the tissues from both Changbai pigs and Ningxiang pigs. The results (figure 3 and table 2) indicated that there were two base variables in exon 1 and one base variable in exon 5 . However, these variations did not cause the alteration of amino acid. The mutation in exon 2 ( $G$ to $A$ ) in $502^{\text {nd }}$ base pairs caused the change of amino acid from Val to Met.

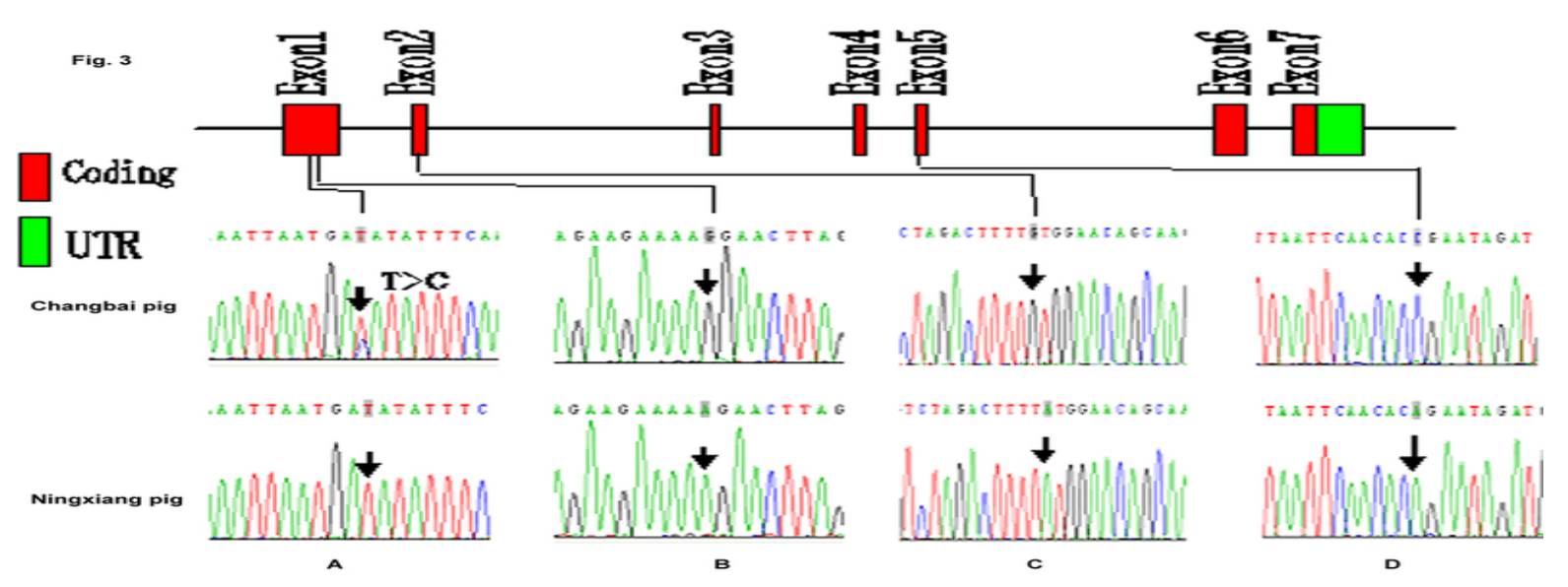

$279 \times 180 \mathrm{~mm}(72 \times 72 \mathrm{DPI})$

Figure 3: ANGPTL3 Exons Sequencing Difference between Changbai Pig and Ningxiang Pig. Genomic structure of the exons which encoding the open reading frame of angpt13 and identified mutations.

Seven exons were all translated (red), and portions of exon 7 was untranslated (Green) in the Angptl3 gene (upper panel). Three different mutations in the angptl3 gene and their sequencing traces were shown at the bottom of the figure (lower panel). (A) The 210th base of the angptl3 ORF; (B) 291st base of the angptl3 ORF; (C) The 502nd base of the angptl3 ORF base base of the angptl3 ORF; (D) The 856th base of the angptl3 ORF. The502nd base variation caused amino variation(Changbai to Ningxiang: Val'!Met). The other three sites were differences in Changbai and Ningxiang pigs, which caused no amino composition difference

\section{Methylation rate variation of ANGPTL3 promoter district}

The ANGPTL3 promotor district BSP-sequencing was used to analyze tissues from both Changbai pigs and Ningxiang pigs. The results showed that: in Changbai pigs, the mean methylation rate was $70.952 \%$, which fatOlean and liver tissue is $75.714 \% 071.429 \%$ and $65.714 \%$ separately; In Ningxiang Pigs, the mean methylation rate was $95.238 \%$, which fat0lean and liver tissue is $98.571 \% 094.286 \%$ and $92.857 \%$ separately (table 3). There are significant difference between Ningxiang and Changbai pigs $(\mathrm{p}<0.05)$.

Table 2: Base variables in ANGPTL3 Exons of Changbai and Ningxing pig

\begin{tabular}{llllll}
\hline Varieties & Tissue & e1-210 & e1-291 & e2-502 & e5-856 \\
\hline Changbai & Fat & $\mathrm{t}(\mathrm{c})$ & $\mathrm{g}$ & $\mathrm{g}$ & $\mathrm{c}$ \\
& Lean & $\mathrm{t}(\mathrm{c})$ & $\mathrm{g}$ & $\mathrm{g}$ & $\mathrm{c}$ \\
& Liver & $\mathrm{t}(\mathrm{c})$ & $\mathrm{g}$ & $\mathrm{g}$ & $\mathrm{c}$ \\
\multirow{4}{*}{ Ningxiang } & Fat & $\mathrm{t}$ & $\mathrm{a}$ & $\mathrm{a}$ & $\mathrm{a}$ \\
& Lean & $\mathrm{t}$ & $\mathrm{a}$ & $\mathrm{a}$ & $\mathrm{a}$ \\
& Liver & $\mathrm{t}$ & $\mathrm{a}$ & $\mathrm{a}$ & $\mathrm{a}$ \\
\hline
\end{tabular}


Table 3: Angpt13 promoter district CpG Island Methylation variation

\begin{tabular}{lllc}
\hline Varieties & Tissue & CpG Methylation rate Mean Methylation rate \\
\hline Changbai & Fat & $75.714 \%$ & $70.952 \%$ \\
& Lean & $71.429 \%$ & \\
& Liver & $65.714 \%$ & $95.238 \%$ \\
\multirow{2}{*}{ Ningxiang } & Fat & $98.571 \%$ & \\
& Lean & $94.286 \%$ & \\
& Liver & $92.857 \%$ & \\
\hline
\end{tabular}

HRM analysis for ANGPTL3 Promoter district Methylation rate

HRM analysis was used to determine the methylation rate of the tissues. The values range from 60 to $80 \%$ in Changbai pigs; In Ningxiang pigs, the methylation rate were between $80-100 \%$; There aresignificant difference between Changbai and Ningxiang pigs $(\mathrm{p}<0.05$, figure 4$)$.

\begin{tabular}{|l|l|}
\hline \multicolumn{2}{|c|}{ Color Methylation Rate } \\
\hline & $100 \%$ \\
\hline & $100 \%$ \\
\hline & $80 \%$ \\
\hline & $80 \%$ \\
\hline & $60 \%$ \\
\hline & $60 \%$ \\
\hline & $40 \%$ \\
\hline & $40 \%$ \\
\hline & $20 \%$ \\
\hline & $20 \%$ \\
\hline & $10 \%$ \\
\hline & $10 \%$ \\
\hline
\end{tabular}

(a)

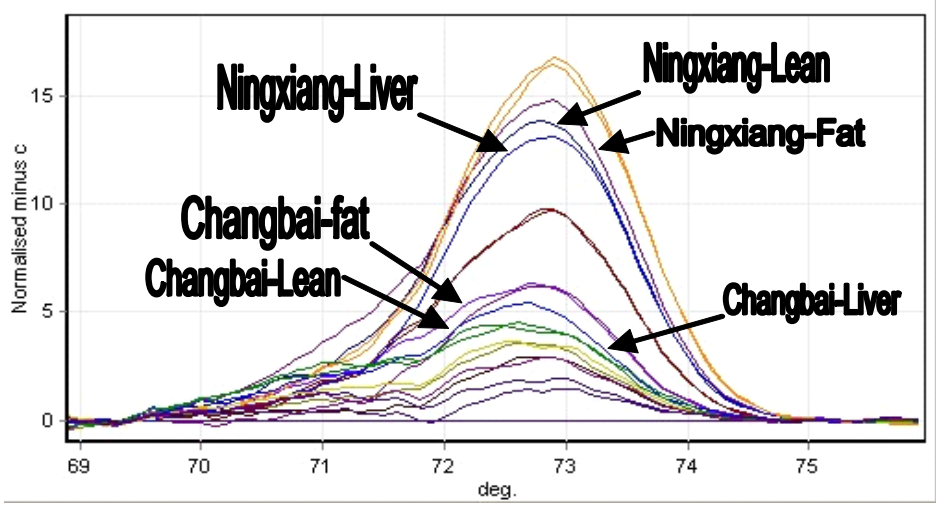

(b)

$278 \times 164 \mathrm{~mm}(72 \times 72 \mathrm{DPI})$

Figure 4: HRM Analysis of ANGPTL3 Promoter district methylation rate

(A) The Standard Curve's Methylation rate were: 100\%, 80\%, 60\%, 40\%, 20\%, 10\%, 0\%. (B) Normalised minus c: Standard Curve didn't point out using arrow( $\mathbf{L}$ ); In lean-type, the lean-fat, lean-lean, lean-liver's methylation rate were $60-80 \%(\mathbf{L})$; In Fat-type: the Fat-fat, Fat-lean, Fat-liver's methylation rate were 80$100 \%$ ( $)$; There are significant difference between Lean-type and Fat-type $(p<0.05)$.

\section{Discussion}

Single nucleotide polymorphism (e.g. exon base mutations) is commonly used as a genomic mapping of genetic markers, and can affect gene function. The experiment performed by Note has shown that there are some nonsense and/or missense mutations in ANGPTL3 gene, and these ANGPTL3 gene mutations are the main reasons for a combined hypolipidemia phenotype ${ }^{15}$. The truncated protein of 122 residues due to the deletion of $5 \mathrm{bp}$ in codon 121 of ANGPTL3 is highly associated with combined hypolipidemia ${ }^{16}$. The rs11207997 polymorphism of ANGPTL3 is demonstrated to be associated with lower plasma HDL-cholesterol and apolipoprotein A-I levels in both adolescents and adults ${ }^{17}$. The ANGPTL4 rs4076317 polymorphism is associated with a higher percentage of body fat in adolescents and a higher waist-to-hip ratio in adults. In our present study, we found that there were four base variations between lean-type pig and fat-type pig in ANGPTL3 exons. Although our study has limitations in the small sample size, it provides important and novel information for us. Whether these ANGPTL3 exon variations can 
distinguish lean-type Pig (Changbai Pig) from fattype pig (Ningxiang Pig) on a certain extent deserves a further study.

DNA methylation is a kind of epigenetic modification. This process, catalyzed by DNA methyltransferases, needs S-adenosyl-methionine as the methyl donor. 5-methyl cytosine is the only chemical modified base present in eukaryotes $\mathrm{DNA}^{18}$. The $\mathrm{CpG}$ islands are mainly located in the promoter and first exon regions ${ }^{19}$. The gene expression can be regulated through methylation of the promoter and nearby region's CpG islands. In general, the promoter region of the island of $\mathrm{CpG}$ hypermethylation may inhibit gene expression, and then cause the down-regulation of the gene expression ${ }^{20}$.

The lower ANGPTL3 promoter CpG island methylation rate in the Changbai pig's liver resulted in higher ANGPTL3 mRNA level, which agreed with the findings of Dokras ${ }^{20}$. If a sparse $\mathrm{CpG}$ promoter also contained a strong enhancer element, gene transcription may take place even though the $\mathrm{CpG}$ sites are methylated ${ }^{21}$. At present, it is not known whether the promoters of the Ningxiang and Changbai Pig's angptl3 contain strong enhancer elements. Inaba ${ }^{22}$ research results show that the ANGPTL3 gene promoter containing a series of characteristic of transcription factor binding sites, such as CCAAT/enhancer binding protein, glucocorticoid receptor, hepatic nuclear factor and LXR. One or several factors of these proteins difference may cause to the difference of the ANGPTL3 expression. Further study shall be taken to find out the reasons that cause the angptl 3 tissue expression differences in Changbai pig and Ningxiang pig.

\section{Conclusion}

The present studies suggest that complete demethylation of every $\mathrm{CpG}$ site in a promoter is not needed to activate gene expression, in as much as the methylation status of a single $\mathrm{CpG}$ site may be sufficient for altered gene expression.

\section{Acknowledgement}

This work was financially supported in part by National Natural Science Foundation of China [Grant 31072167], Major Program of Science and Technology of Hunan Province (2009FJ1005) and Ministry of Education Innovation Team (Number IRT0963).

\section{References}

1. Preiss-Landl K, Zimmermann R, Hämmerle G, Zechner R. Lipoprotein lipase: the regulation of tissue specific expression and its role in lipid and energy metabolism. Current opinion in lipidology 2002; 13 (5): 471-481.

2. Koster A, Chao YB, Mosior M, et al. Transgenic angiopoietin-like (angptl) 4 overexpression and targeted disruption of angptl4 and angptl3: regulation of triglyceride metabolism. Endocrinology 2005; 146 (11): 4943-4950.

3. Ono M, Shimizugawa T, Shimamura M, et al. Protein region important for regulation of lipid metabolism in angiopoietin-like 3 (ANGPTL3). Journal of Biological Chemistry 2003; 278 (43): 41804-41809.

4. Ge H, Cha JY, Gopal H, et al. Differential regulation and properties of angiopoietin-like proteins 3 and 4. Journal of lipid research 2005; 46 (7): 1484-1490.

5. Shimizugawa T, Ono M, Shimamura M, et al. ANGPTL3 decreases very low density lipoprotein triglyceride clearance by inhibition of lipo-protein lipase. Journal of Biological Chemistry 2002; 277 (37): 33742-33748.

6. Shimamura M, Matsuda M, Kobayashi S, et al. Angiopoietin-like protein 3, a hepatic secretory factor, activates lipolysis in adipocytes. Biochemical and biophysical research communications 2003; 301 (2): 604-609.

7. Shimamura M, Matsuda M, Yasumo H, et al. Angiopoietin-like protein3 regulates plasma HDL cholesterol through suppression of endothelial lipase. Arteriosclerosis, Thrombosis, and Vascular Biology 2007; 27 (2): 366-372.

8. Koishi R, Ando Y, Ono M, et al. Angptl3 regulates lipid metabolism in mice. Nature genetics 2002; 30 (2): 151-157.

9. Xiao HB, Sun ZL, Zhou N. 1, 3, 5, 8Tetrahydroxyxanthone regulates ANGPTL3LPL pathway to lessen the ketosis in mice. European Journal of Pharmaceutical Sciences 2012; 46 (1-2): 26-31.

10. Feng SQ, Chen XD, Xia T, et al. Cloning, chromosome mapping and expression characteristics of porcine ANGPTL3 and -4 . Cytogenetic and genome research 2006; 114 (1): 4449.

11. Newell-Price J, Clark AJL, King P. DNA methylation and silencing of gene expression. Trends in Endocrinology and Metabolism 2000; 11 (4): 142-148. 
12. Attwood JT, Yung RL, Richardson BC. DNA methylation and the regulation of gene transcription. Cellular and molecular life sciences 2002; 59 (2): 241-257.

13. Leonhardt H, Page AW, Weier HU, Bestor TH. A targeting sequence directs DNA methyltransferase to sites of DNA replication in mammalian nuclei. Cell 1992; 71 (5): 865-873.

14. Conklin D, Gilbertson D, Taft DW, et al. Identification of a mammalian angiopoietinrelated protein expressed specifically in liver. Genomics 1999; 62 (3): 477-482.

15. Noto D, Cefalu AB, Valenti V, et al. Prevalence of ANGPTL3 and APOB gene mutations in subjects with combined hypolipidemia. Arteriosclerosis, Thrombosis, and Vascular Biology 2012; 32 (3): 805-809.

16. Martin-Campos JM, Roig R, Mayoral C, et al. Identification of a novel mutation in the ANGPTL3 gene in two families diagnosed of familial hypobetalipoproteinemia without APOB mutation. Clinica Chimica Acta 2012; 413 (5-6): 552-555.

17. Legry V, Bokor S, Cottel D, et al. Associations between common genetic polymorphisms in angiopoietin-like proteins 3 and 4 and lipid metabolism and adiposity in European adolescents and adults. Journal of Clinical Endocrinology and Metabolism 2009; 94 (12): 50705077.

18. Feltus FA, Lee EK, Costello JF, Plass C, Vertino PM. Predicting aberrant $\mathrm{CpG}$ island methylation. Proceedings of the National Academy of Sciences 2003; 100 (21): 12253-12258.

19. Marsit CJ, Houseman EA, Christensen BC, et al. Examination of a $\mathrm{CpG}$ island methylator phenotype and implications of methylation profiles in solid tumors. Cancer research 2006; 66 (21): 10621-10629.

20. Dokras A, Coffin J, Field L, et al. Epigenetic regulation of maspin expression in the human placenta. Molecular buman reproduction 2006; 12 (10): 611-617.

21. Bird A. The essentials of DNA methylation. Cell 1992; 70 (1): 5.

22. Inaba T, Matsuda M, Shimamura M, et al. Angiopoietin-like protein 3 mediates hypertriglyceridemia induced by the liver X receptor. Journal of Biological Chemistry 2003; 278 (24): 21344-21351. 